\title{
Ingegneri militari nella Sicilia degli Asburgo: formazione, competenze e carriera di una figura professionale tra Cinque e Seicento
}

\section{Maurizio Vesco}

Dipartimento di Architettura - Università degli Studi di Palermo, Palermo, Italia, mauriziovesco@gmail.com

\begin{abstract}
During the Sixteenth century and the first half of the following one, Sicily played a key role in the strategy of defense of the Spanish Empire from the looming Turkish threat, being an island considered as a baluarte against the Turks. Yet despite this absolute centrality in the military policy of the Spanish monarchy, till now historical research has preferred to provide broader spectrum interpretations at the expense of in-depth studies on individual technicians or overall pictures about the figure of the military engineer. We will present a synoptic view attesting the time period of activity covered by each military technician working for the kingdom of Sicily, within a wider study concerning the origin of this professional figure, the diversification of technicians working on fortifications, the progressive changes in the organizational chart and the enrichment of competences for military engineers much beyond l'arte del fortificare.
\end{abstract}

Keywords: Military Engineers, Sicily, Hapsburgs.

\section{Introduzione}

La Sicilia, nel XVI secolo e per almeno la prima metà del successivo, svolse un ruolo chiave nella strategia della difesa dell'Impero spagnolo dalla incombente minaccia turca, un'isola definita già ai tempi come baluarte nei confronti del nemico ottomano. Eppure, nonostante questa assoluta centralità nella politica militare della monarchia asburgica, che egualmente caratterizzò l'isola nella fase "espansionistica" degli avamposti africani, la ricerca storiografica ha preferito fornire letture di ampio spettro sulle campagne fortificatorie, alcune delle quali attuali ancor'oggi (Giuffré, 1976), piuttosto che studi monografici sui singoli ingegneri militari o di inquadramento generale di questa figura professionale: il suo ruolo, il processo di selezione e reclutamento, le competenze e le modalità di esercizio della professione, la messa a punto della figura istituzionale nell'ambito dell'organigramma “statale". L'esiguità di contributi scientifici di questo ultimo tipo, nonostante alcuni interessanti contributi (Di Fede, 1998), fa sì che le zone d'ombra rimangano ancora preponderanti, persino riguardo alla semplice successione negli incarichi, tanto che sino ad oggi un quadro sinottico che attesti gli archi temporali coperti dai diversi ingegneri militari in servizio nel Regno di Sicilia è ancora mancante e proveremo a presentarlo, seppur limitatamente al XVI secolo, in questa occasione (Fig. 1).

\section{Origine di una figura professionale}

Nell'aprile del 1523 il viceré di Sicilia Ettore Pignatelli, conte di Monteleone, figura chiave della strategia politico-militare di Carlo V 


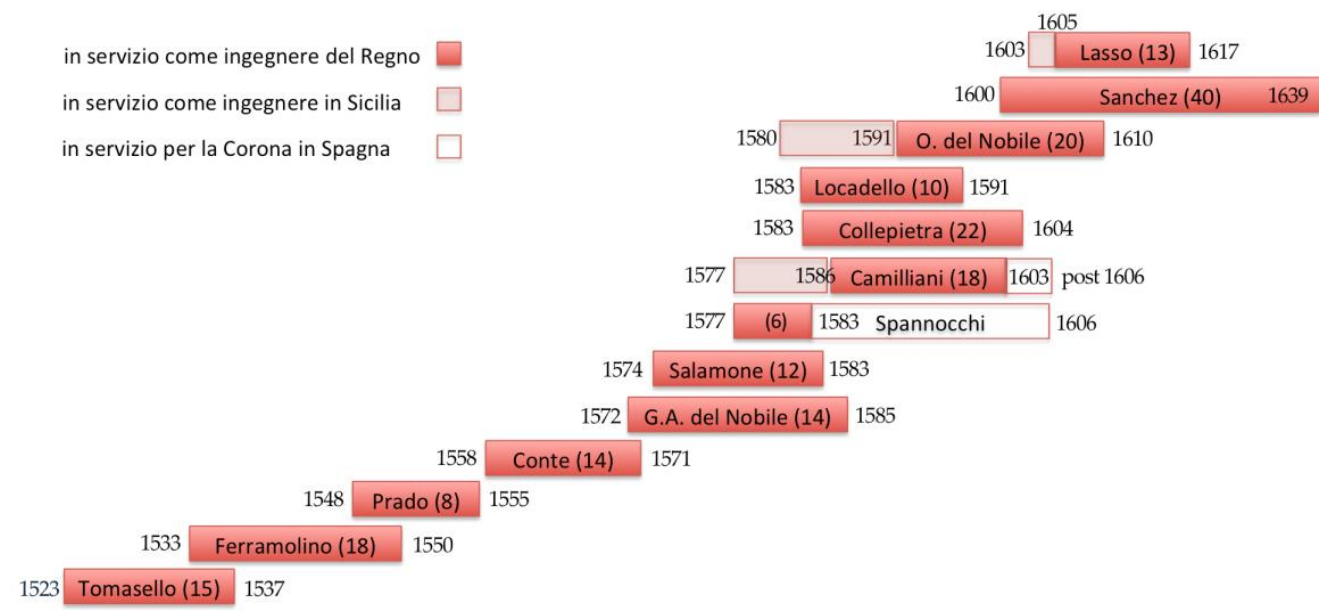

Fig. 1 - Tavola sinottica del periodo di attività degli ingegneri del Regno di Sicilia nel XVI secolo.

nell'isola, dava incarico a Pietro Antonio Tomasello, magistro ingigneri dila cità di Padua, di attendere ai grandi progetti di riforma e ammodernamento delle difese delle città costiere siciliane, secondo quanto già ordinato dal sovrano tre anni prima (Vesco, 2010).

Se a primo acchito potrebbe sembrare che la nomina dell'ingegnere fosse strettamente connessa alla esecuzione di singole, specifiche opere edificatorie, secondo un modus operandi attestato nell'isola tra fine Quattrocento e primi anni del secolo successivo, già l'anno seguente essa rivela la sua vera finalità: l'introduzione nel più articolato organigramma statale di una nuova figura istituzionale, quella dell'ingegnere del Regno.

Parrebbe, infatti, che fino a quel momento della progettazione delle fortificazioni, o quanto meno dei progetti più delicati e complessi, fosse stato dato incarico a consulenti esterni, chiamati a intervenire ad hoc e a fornire pareri, espressi individualmente o insieme ad altri tecnici riuniti in apposite consulte, secondo una prassi che sarebbe stata adottata ancora in talune occasioni nei decenni a venire. Avvenne così per il catalano Baldar Metall, maestro d'artiglieria, che nel giugno del 1485 si imbarcò da Barcellona alla volta di Palermo, con l'incarico di redigere il progetto per la cittadella del Castellammare della capitale siciliana (Gaeta, 2010). Fu lo stesso, alcuni decenni più tardi, per il nobilis
Antonello da Trani, capitano d'artiglieria al servizio del Regno di Napoli, che nel 1518 venne inviato sull'isola sia per elaborare un nuovo piano per la stessa cittadella, sia per realizzare altri otto disegni di progetto di non ben precisate fortezze del Regno (Vesco, 2014).

È dunque proprio con l'arrivo in Sicilia dal Veneto di Pietro Antonio Tomasello, assai probabilmente allievo di Gabriele Tadino, che viene di fatto istituita la carica dell'ingegnere del Regno. Per lungo tempo tale incarico rimase appannaggio di una sola persona, alla quale però, in rari casi, poteva essere affiancato un altro tecnico, a cui veniva corrisposto regolarmente un salario e che poteva anch'egli ricoprire la carica di ingegnere regio. Fu così, ad esempio, nel 1533 quando a Tomasello, gravemente ammalato e già avanti negli anni, venne affiancato il più giovane Antonio Ferramolino da Bergamo. Il viceré Pignatelli, approfittando del passaggio in Sicilia dell'ingegnere in fuga da Corone caduta in mano turca, città in cui era stato inviato dal sovrano per redigere il progetto di fortificazione dell'importante avamposto greco, gli richiese un parere sui piani che si stavano portando avanti sull'isola (Tadini, 1977). Ferramolino, come è noto, non sarebbe più ripartito: i due ingegneri veneti si sarebbero infatti divisi tra loro i cantieri siciliani ed entrambi avrebbero ricoperto per quattro anni, fino alla morte di Tomasello, nel 
1537, l'incarico istituzionale con un eguale compenso di 300 ducati d'oro (Vesco, 2010).

Alla morte di Ferramolino, occorsa il 18 agosto del 1550 durante l'assedio di Africa, l'odierna Mahadia, questi, rimasto dal 1537 da solo a ricoprire quel ruolo, sarebbe stato rimpiazzato da Pedro Prado, chiamato già due anni prima in Sicilia da Napoli, dove quasi certamente era stato al seguito del celebre ingegnere Luis Escrivà.

Dopo la morte di Prado, avvenuta nei primi mesi del 1555, ad essere nominato fu Antonio Conte, giunto al seguito del viceré don Juan de la Cerda, duca di Medinaceli, con il quale prese parte alla sfortunata impresa di Tripoli, nel marzo del 1560, approntando un forte in terra e altre opere campali in occasione della battaglia delle Gerbe (de Ulloa, 1566). Se fino ad adesso quasi nulla si sapeva dell'entrata in servizio di Conte, nostre acquisizioni documentarie fanno risalire il suo arrivo in Sicilia al 1558 e comprovano la sua nomina nel settembre dell'anno successivo, venendo scelto «per essere soldato platico et persona di experiencia et di qualità nel detto suo cargho di ingegnero» (ASPa, C, reg. 391, c. 288v). Il Conte, come dimostrano i nuovi documenti, riuscì a scampare alla morte nella tragica disfatta delle Gerbe, cadendo, però, prigioniero dei Turchi insieme a centinaia di altri commilitoni e riuscendo a sottrarsi alla prigionia dietro pagamento di un cospicuo riscatto. Sarebbe stato meno fortunato dieci anni più tardi, quando, nel 1571, il suo nome andò ad ingrossare il lungo elenco dei caduti cristiani nella battaglia navale di Lepanto (Mazzarella, Zanca, 1985).

Ancora negli anni Sessanta del Cinquecento la figura dell'ingegnere regio non era forse ancora del tutto normata nell'ambito dell'organigramma statale, dato il persistere di una certa variabilità, se non arbitrarietà, nei processi di selezione e nel numero di tecnici assoldati. Ad esempio, riteniamo che per alcuni anni, dopo la morte di Prado, la plaza di ingegnere sia rimasta vacante. Lo proverebbe l'incarico conferito nel 1556 dal viceré Juan de Vega al maestro Bartolomeo Cascone, o meglio Guascone, forse di origine continentale e pirenaica quindi, per occuparsi delle difese di Messina, Trapani e probabilmente della fortificazione dell'avamposto di Pantelleria (ASPa, TRP, LV, reg. 425, cc. 144r). D'altronde, anche il successore di Vega, il duca di Medinaceli, si sarebbe avvalso delle competenze di Guascone per opere alle mura palermitane (ASCP, ABP, c. 103v.) e lo avrebbe elevato nel 1558 al ruolo di revisore e misuratore di fabbriche e ponti, figura forse introdotta $a d$ hoc dopo la nomina di Conte a ingegnere, che inevitabilmente scalzava l'abile capomastro, a suo tempo scelto in quanto «persona platica et experta in li disigni et modelli deli detti fabrici et fortificationi di esso Regno et di essi ponti» (ASPa, TRP, LV, reg. 435, c. $368 \mathrm{v}$ ); si introduceva così una nuova figura complementare, certo subordinata a quella dell'ingegnere, come comproverebbe anche il minore salario corrisposto.

L'ambiguità di questo profilo, almeno nella fase iniziale, è confermato da quanto avvenuto alla morte di Guascone: nel 1568, infatti, in tre si fecero avanti per ricoprirne l'incarico. Si trattava di due affermati capomastri, il piemontese Giovan Francesco Lombardo, già attivo per la Compagnia di Gesù e in molti importanti cantieri di palazzi cittadini, nonchè possessore di libri e trattati di architettura, Vincenzo Pernaci, «architecturi et mastro di scultura», nonché lo spagnolo Miguel de Medina, di cui nulla al momento è noto, che sarebbe stato preferito agli altri due (ASPa, TRP, M, reg. 148, c. 69r), venendo nominato revisore nel febbraio dell'anno successivo (ASPa, TRP, LV, reg. 547, c. 45r). È interessante osservare come Medina poteva, comunque, fregiarsi del titolo di ingegniero (ASPa, TRP, LV, reg. 535, c. 252r), a riprova di una distinzione tra le figure assai labile e soggetta a differenti interpretazioni.

\section{La dura vita dell'ingegnere militare}

Per meglio comprendere la figura dell'ingegnere militare, le molte sfaccettature della personalità dei protagonisti di quella stagione epica che avrebbe condotto alla costruzione di complessi sistemi fortificatori e di mastodontiche macchine da guerra, fatte di terra e calce, di pietra e fascine, opere che avrebbero profondamente 
segnato, sino all'alba dell'età contemporanea, l'immagine delle città siciliane e indotto sovente l'alterazione dell'orografia del territorio, non si puó non tenere conto delle condizioni di difficoltà, se non di vero e proprio pericolo, in cui i tecnici erano costretti a svolgere la loro attività, assolvendo a carichi di lavoro spesso immani.

Obbligati a spostarsi di continuo da un capo all'altro dell'isola, ma sovente anche extra regnum, erano esposti in prima persona a rischi altissimi, legati non soltanto alle più delicate missioni al fronte a cui erano tenuti -si pensi alla già ricordata tragica fine di Ferramolino e Conte-, ma anche al viaggiare per terra e per mare. Fu così, ad esempio, per Tomasello che nel 1526, inviato da Siracusa a Malta, venne intercettato nelle acque del Mediterraneo dai Turchi e, fatto prigioniero, venne liberato solo dopo lunghe trattative (Vesco, 2010). Similmente va ricordato, anche a testimonianza di un territorio insicuro e malamente controllato dalle autorità, come nel 1534 Ferramolino venne rimborsato dalla Corte per il cavallo, i vestiti e gli altri beni personali che gli erano stati sottratti da banditi durante il suo viaggio da Palermo verso Siracusa e Augusta.

Riteniamo che sia stato proprio l'enorme carico di lavoro gravante sull'ingegnere del Regno, incarico ricoperto da una sola persona praticamente per quasi un cinquantennio, a indurre più tardi la Corte a moltiplicare stabilmente i posti, giungendo a definire quelle tre plazas che saranno previste dall'ultimo quarto del Cinquecento in poi. Troppi cantieri, troppo lunghe distanze da coprire, ma anche una sempre maggiore varietà di incarichi rendevano di fatto impossibile fare affidamento su un solo tecnico.

Le modalità di esercizio della professione, d'altra parte, rendevano assai dura la vita dell'ingegnere militare che continuava, sul solco di una tradizione tutta quattrocentesca, a rimanere uomo d'arme e in quanto tale privo il più delle volte di una vita privata, di una famiglia, uomini legati soltanto a un fratello o a una sorella lontani, rimasti nei luoghi d'origine, ricordati nei testamenti redatti prima di andare in battaglia o di un viaggio o all'insorgere della malattia, come comprovato dal testamento redatto nel 1528 dal padovano Tomasello (ASTp, ND, G. De Simone, a. 1521, 1.6.1528). In altri casi, come nelle ultime volontà del giovane Ferramolino dettate nel 1538, non vi era più traccia di alcun legame familiare e a essere ricordati erano solo $\mathrm{i}$ due fedeli servitori Domenico di Siena e Giovanni di Roma, nonché come erede il conservatore del Regio Patrimonio Peri Andrea Lombardo, l'alto funzionario in cui l'ingegnere nella solitudine dei giorni siciliani doveva aver trovato anche un amico (ASPa, ND, St. I, vol. 2921, 17.2.1538).

D'altronde, l'unione con una donna, il procreare dei figli, causava non poche interferenze con lo svolgimento di un mestiere assai impegnativo come quello: fu proprio la scelta di contrarre matrimonio ad avere, ad esempio, segnato il declino dell'attività professionale di Antonio Ferramolino. La volontà di risiedere stabilmente con la famiglia, la necessità di assicurare, una volta giunta la prole, un maggiore reddito familiare e forse persino un'attività da lasciare ai figli, lo convinse a dar vita, probabilmente insieme alla moglie Eleonora, a un attività imprenditoriale, prendendo in appalto prima il lucroso cantiere delle mura di Catania e poi quello della torre del caricatore di Agrigento, scelta che, come prevedibile, avrebbe generato da subito grossi conflitti di interesse, finendo con il mettere l'ingegnere bergamasco in cattiva luce agli occhi della Corte e dell'implacabile Vega (Aricò, 2012).

D'altronde, in altri casi, la Corte avrebbe accettato simili commistioni, consentendo che in una sola persona si riunissero funzionario governativo preposto al controllo e fornitore di beni e servizi: ad esempio, l'ingegnere Giovanni Antonio del Nobile, sin dal 1563 (ASPa, TRP, $\mathrm{LV}$, reg. 560 , c. $22 \mathrm{v}$ ), un decennio prima di assumere l'incarico dunque, avrebbe iniziato un'attività di produzione del salnitro per la polvere da sparo e di pezzi e complementi di artiglieria che avrebbe proseguito regolarmente anche dopo la nomina, grazie a quella concessione di tutte le miniere del Regno, di certo assai redditizia, rinnovatagli dall'autorità 
vicereale ancora nel 1582 (ASPa, TRP, M, reg. 245 , c. 392 r).

È ormai assodato, comunque, che i cantieri per le fortificazioni, che impiegavano enormi risorse finanziarie per la fornitura di manodopera specializzata e non, di materiali di ogni genere, per l'esecuzione di lavorazioni anche complesse -in primo luogo palificate, fondazioni idrauliche e interri per la modifica della linea di costa-, erano occasione propizia per malversazioni e truffe alla Corte, da cui non sempre gli ingegneri del Regno rimanevano estranei. Segnaliamo, a questo proposito, come il celebre scultorearchitetto toscano Camillo Camilliani, in servizio per il Regno di Sicilia in qualità di ingegnere regio dal 1577 (Scarlata, 1992), nel 1606, mentre era in servizio in Spagna (Bosch, 2014), venne incriminato per truffa dalla Corte di Madrid su richiesta delle autorità siciliane: «este hombre ha procedido mal y debria ser castigado por el delicto que cometio en ciertas fabricas de fortificaciones que se le encomendaron en la Licata, en que llevò injustamente de la Corte buena suma de dinero» (AGS, CP, lib. 749, c. 96r).

È pur vero, che alcuni tecnici, in particolare nella seconda metà del XVI secolo, sarebbero riusciti ad acquisire uno status sociale elevato, garantendo la costruzione di una solida rete di appoggi familiari attraverso consanguinei $\mathrm{e}$ discendenti, posti a occupare importanti uffici pubblici: fu il caso, ad esempio, dell'ingegnere Vincenzo Locadello, capitano d'armi attivo sul finire del secolo, il cui figlio Lazzaro divenne capitaneus ordinarius Sue Catholice Magestatis, mentre il fratello Francesco ricopriva già l'incarico di maestro razionale del Regno, una delle cariche più alte dell'amministrazione isolana, condizione che crediamo possa avere favorito non poco la carriera dell'ingegnere.

\section{Oltre l'arte del fortificare: le competenze in architettura degli ingegneri regi}

Se è da ritenere scontata la perizia degli ingegneri del Regno nel campo dell'architettura militare, ivi comprese le tecniche dell'artiglieria e della costruzione di difese in terra per lo scontro diretto sul campo di battaglia, meno immediata è la competenza che questi, in taluni casi, potevano vantare anche nell'architettura civile e religiosa, o persino nella progettazione urbana.

Eppure non pochi degli ingegneri del Regno di Sicilia furono impegnati in questi ambiti progettuali. Se il fenomeno ci è noto a partire dalla metà del Cinquecento con l'entrata in scena di Pedro Prado, questo sembra registrare l'acme tra la fine del secolo e gli inizi del successivo. Proprio Prado sembra qualificarsi come un ingegnere del Regno un pò fuori dall'ordinario. È stato, infatti, dimostrato come il tecnico spagnolo non solo giunse in Sicilia nel 1548, due anni prima della morte del Ferramolino dunque, quando il bergamasco ricopriva l'unica plaza a quel tempo disponibile di ingegnere regio, ma soprattutto che era stato chiamato da Vega appositamente per occuparsi del progetto di riforma del Palazzo Reale di Messina (Aricò, 2014).

Prado dunque architetto prima ancora che ingegnere militare. Dovrebbe sempre essere, così, ricondotta a questa sua peculiare identità sia il raffinato progetto per la porta Vega o Africa, costruita a Palermo per celebrare la vittoria del viceré a Mahadia, raffinata espressione di un linguaggio architettonico aggiornato che guarda compiaciuto al classicismo (Vesco, 2013), sia il piano urbanistico per la nuova città-fortezza di Carlentini, la moderna piazzaforte fondata in onore dell'imperatore, ispirato a una matura cultura di matrice polibiana (Aricò, 2012).

Inoltre, gli ingegneri regi -certamente Prado, Salamone, Camilliani- vennero coinvolti, sotto il governo di Vega prima e di Marco Antonio Colonna poi, nella progettazione di una nuova rete di ponti per il potenziamento dei collegamenti tra le diverse parti dell'isola e ciò non solo per ragioni militari.

\subsection{Per la difesa e la messa in sicurezza del territorio}

È sempre a partire dalla seconda metà del Cinquecento che l'ingegnere regio viene anche preposto alla difesa e alla messa in sicurezza del 
territorio siciliano, da sempre esposto a dissesti idro-geologici: frane, alluvioni, inondazioni.

Siamo dell'avviso che in questo senso potrebbero essere stati i fatti tragici della china di Palermo, la catastrofica alluvione che si abbattè sulla città nel 1557 (Vesco, 2014b), a suscitare una nuova, seppur larvale, attenzione ai problemi legati al dissesto idrogeologico e al controllo delle acque, o quanto meno a generare una prima consapevolezza che la risoluzione di simili problematiche dovesse essere affidata a tecnici abili e specializzati, in primo luogo agli ingegneri del Regno. Lo conferma quanto avvenne nel 1593, in occasione di un nuovo disastro: quando una frana, causata da piogge alluvionali e dalla esondazione di fiumi, colpì la città di Corleone, provocando la distruzione di uno dei suoi borghi, la Regia Corte inviò l'ingegnere Camilliani per la messa in sicurezza dell'abitato (ASPa, TRP, M, reg. 452, c. 97r).

Ma il problema del controllo dei corsi d'acqua, attuato mediante il loro imbrigliamento con argini e muraglioni o nei casi più complessi con la deviazione dei fiumi in nuovi letti, sarebbe rimasto centrale ancora nei primi del Seicento: ad esempio, in area peloritana, solcata da una serie di fiumare e da sempre esposta al rischio alluvionale, nel 1607 venne inviato l'ingegnere alemanno (ma forse solo ticinese) Orazio del Nobile, per approntare nuove soluzioni a difesa del territorio (ASPa, TRP, Co, reg. 17, c. 106r).

\subsection{Architettura e progetto urbano}

Abbiamo già accennato a come Prado diede prova nella progettazione dell'impianto urbano di Carlentini, della sua vasta cultura progettuale e della conoscenza, nello specifico, della trattatistica e del dibattito contemporaneo sulla castrametatio, da Polibio a Machiavelli e ai Commentarii di Cesare, da lì a poco riccamente illustrati dalle tavole di Palladio. Va ricordato che sempre Prado negli stessi anni fu impegnato, su incarico del viceré spagnolo, nell'unica vera esperienza di addizione urbana che la capitale del Regno abbia conosciuto nella prima età moderna: il piano di espansione del quartiere della Kalsa (Vesco, 2013). Questo era correlato al potenziamento delle difese verso mare e in particolare alla "correzione" di quella gravissima debolezza che ancora sino al 1551 il tracciato del circuito murario cittadino presentava, nonostante il precedente piano elaborato da Ferramolino, "correzione" che tra l'altro avrebbe perfezionato quella forma urbis quadrata e quadrifida di Palermo presto oggetto di innumerevoli speculazioni filosofico-religiose.

Sempre sul piano della progettazione urbana, quello di Prado non fu un caso isolato tra gli ingegneri del Regno di Sicilia. Trent'anni più tardi Orazio del Nobile, in servizio dal $1591 \mathrm{ma}$ già da molti anni impiegato dalla Corte nel ruolo specifico di ingegnere delle fabbriche $\mathrm{di}$ Milazzo, con un campo d'azione dunque limitato ad un solo cantiere, nel 1585 fu impegnato nella pianificazione della nuova espansione della città peloritana, nell'area ai piedi della collina fortificata, sovraintendendo personalmente alle operazioni di tracciamento al suolo di strade, piazze e isolati (ASPa, TRP, M., reg. 245, c. $119 \mathrm{v})$. D'altro canto, agli ingegneri regi si sarebbe fatto sovente ricorso anche per interventi urbanistici a più piccola scala, quale ad esempio l'apertura di una nuova strada, soprattutto quando si trattava di interventi delicati da un punto di vista tecnico-strutturale o per la difficoltà nella gestione e nella organizzazione del cantiere. Nell'anno 1600 sempre del Nobile, forse coadiuvato dall'ingegnere regio Giovan Battista Collepietra, uomo a quella data di più esperienza e autorevolezza, avrebbe attuato e sovrainteso a Palermo al tracciamento della celebre Strada Nova o Maqueda (Fanelli, 1998), attuato con una vasta ma controllatissima campagna di demolizioni nel tessuto fitto della capitale siciliana, uno sventramento «che quantitativamente non trova equivalenti nell'Europa del '500» (Fagiolo, 1981). Prima di lui, il già ricordato ingegnere Medina, sempre a Palermo, per volontà del duca di Medinaceli aveva redatto, nel 1561 , ben sei anni prima dell'avvio del cantiere della strada Toledo, il progetto per uno dei più lunghi e moderni rettifili non solo italiani, la strada più tardi indicata come via di Porta di Castro, a una estremità della quale aveva previsto una piazza quadrata dal carattere monumentale e celebrativo (Vesco, 2010b). 
È ben noto, poi, come molti ingegneri del regno ricevettero anche l'incarico della progettazione di importanti fabbriche regie, in primo luogo dei palazzi reali: fu così per Giovanni Antonio Salamone e Giovan Battista Collepietra nel Sacrum Regium Palacium di Palermo, ad esempio, ai quali più tardi sarebbe succeduto, sempre nella medesima fabbrica, questa volta per la realizzazione del cortile e dello scalone, l'ingegnere Diego Sanchez (Di Fede, 2000).

Ma gli ingegneri regi si sarebbero confrontati anche con i temi dell'architettura religiosa. Ad esempio Prado, già autore nel 1547 della perduta cappella del forte Sant'Elmo di Napoli (De Castro, Cobos, 2000), avrebbe progettato la chiesa Madre della nuova civitas carleontina (Aricò, 2012), mentre Orazio del Nobile più tardi, nel 1604, la chiesa della Concezione a Palermo (Filizzola, 1967). Inoltre, nuovi documenti attestano come un primo progetto per la ricostruzione della chiesa di santa Eulalia dei Catalani, sempre a Palermo, vada ricondotto anch'esso al toscano Collepietra, in servizio dal 1583. Nel settembre del 1585 l'ebanista Agostino Castello si allogava a uno dei deputati della fabbrica del nuovo tempio per realizzarne il modello ligneo. Si stabiliva «che il modello si habia di fare cum tucti quilli parti et minutie che nelli desegni fatti si notano et occurrendo adjungere o levare de decti disegni per meglio perfectione alcuna cosa sia licito al magnifico Joanne Bactista Collipetra, uno delli regii ingigneri de quisto regno, como autore de decto desegno»; 1'opera lignea avrebbe dovuto in tutto adeguarsi ai disegni dell'ingegnere «acciò decto modello possa veramente essere luce et guida de quanto si havirà da fare in essa fabrica» (ASPa, ND, st. I, vol. 5257, c. 39r).

In conclusione, se in una prima fase sono $\mathrm{i}$ viceré a richiedere alla Corona l'invio di tecnici specializzati in Sicilia o a proporre a personaggi in transito nell'isola di risiedervi stabilmente assumendo la carica di ingegnere del Regno, sul finire del Cinquecento, in virtù di un ambiente isolano assai mutato, saranno al contrario tecnici già presenti e forse operanti in Sicilia a proporsi alla Corte per ricoprire tale ruolo, avvalendosi quando possibile o di legami di consanguineità fu così per Orazio del Nobile nominato dal viceré conte Alba de Liste alla morte del fratello, l'ingegnere maggiore Giovanni Antonio (AGS, SP, lib. 959, c. 73r)-, o, ancora meglio, della buona parola del viceré in persona. In taluni casi si ricorse persino allo stesso sovrano, come accadde per Giulio Lasso che nel 1605, dopo la sfortunata esperienza delle fortificazioni di Bretagna (Cámara, 2011), ricorse a Filippo III chiedendo di essere assunto in Sicilia poiché «vacan al presente tres plaças de Ingenieros del dicho reyno, las dos por muertes de Rafael Locadelo y Juan Baptista Colipetro y la otra por ausencia de Camilo Camiliani», non senza aver prima sottolineato la sua competenza «en cosas de fortificacion, matematica y architectura militar» e rammentato i suoi meriti nella difesa di Messina in occasione di un attacco turco alla costa calabra (AGS, DP, lib. 865, c. 86r). Tre plazas, dunque, che però già l'anno successivo sembrano essere diventate quattro se il viceré duca di Feria, davanti alle richieste del sovrano di inviare in Spagna dalla Sicilia qualche buon ingegnere militare, gli rispondeva che «en este reyno no ay ingeniero tolerable quanto mas eminente como Vuestra Magestad le quiere, aunque aqui ay quatro que llevan plaça y si tien falta de mejores, yo no tengo noticia de ninguno; en Flandes es de creer que havrà deste hombres pues las artes valen adonde ay exercicio dellas» (AGS, Estado, Sicilia, leg. 1162/80). La Sicilia, con la poca perizia dei suoi ingegneri militari e la scarsa versatilità nell'arte della guerra, pagava così lo scotto di essere da anni lontana, fortunatamente, dalle trincee e dai campi di battaglia.

\section{Note}

The research leading to these results has received funding from the ERC under the EU's Seventh Framework Programme (FP7/20072013) / ERC grant agreement $n^{\circ} 295960$ COSMED. ABP: Atti, bandi e provviste; AGS: Archivo General Simancas; ASCP: Archivio Storico Comunale Palermo; ASPa: Archivio di Stato Palermo; ASTp: Archivio di Stato Trapani; C: Cancelleria; Co: Consulte; CP: Consultas de Partes; DP: Dispachos de Partes; LV: Lettere Viceregie; M: Memoriali; ND: Notai defunti; TRP: Tribunale Real Patrimonio. 


\section{References}

De Ulloa A. (1566). La Historia dell'impresa di Tripoli di Barbaria. Francesco Rampazetto. Venezia f. $16 r$.

Filizzola C. (1967). La chiesa dell'Immacolata Concezione di Maria Vergine. S. Andò \& figli. Palermo. Giuffré M. (1976). "Palermo «città murata» dal XVI al XIX secolo". in Quaderno dell'Istituto Dipartimentale di Architettura ed Urbanistica dell'Università di Catania-8. Vito Cavallotto Editore. Catania. pp. 41-68.

Tadini G. (1977). Ferramolino da Bergamo. L'ingegnere militare che nel '500 fortificò la Sicilia. Poligrafiche Bolis. Bergamo. pp. 27-33.

Fagiolo M., Madonna M.L. (1981). Il Teatro del Sole. La rifondazione di Palermo nel Cinquecento e l'idea della città barocca. Officina Edizioni. Roma. p. 47.

Mazzarella S., Zanca R. (1985). Il libro delle torri. Le torri costiere di Sicilia nei secoli XVI-XX. Sellerio. Palermo. p. 104.

Scarlata M. (1992). L'opera di Camillo Camilliani. Istituto Poligrafico e Zecca dello Stato. Roma.

Di Fede M.S. (1998). "La gestione dell'architettura civile e militare a Palermo tra XVI e XVII secolo: gli ingegneri del regno". in Espacio, Tiempo y Forma, VII, Historia del Arte, 11 (1998). pp. 135 153.

Fanelli G. (1998). I Quattro Canti di Palermo. Il cantiere barocco nella cultura architettonica ed urbanistica della capitale vicereale. Regione siciliana. Palermo.

De Castro Fernandez J.J., Cobos Guerra C. (2000). "El debate en las fortificaciones del imperio y la monarquía española 1535-1574". in Hernando Sanchez C.J. (coord.). Las fortificaciones de Carlos $V$. Ediciones del Umbral. Madrid. pp. 254, 262.

Di Fede M.S. (2000). Il Palazzo Reale di Palermo tra XVI e XVII secolo. Medina. Palermo.

Gaeta A. (2010). "A tutela et defensa di quisto regno". Il castello a mare di Palermo, Baldiri Meteli e le fortificazioni regie in sicilia nell'età di Ferdinando il Cattolico. Qanat. Palermo. pp. 99-101.

Vesco M. (2010). "Pietro Antonio Tomasello de Padua: un ingeniero militar véneto en la Sicilia de Carlos V". in Espacio, Tiempo y Forma - Revista de la Facultad de Geografía e Historia de la UNED de Madrid», VII, Historia del Arte, 22-23 (2009-2010). pp. 45-73.

Vesco M. (2010b). Viridaria e città. Lottizzazioni a Palermo nel Cinquecento. Edizioni Kappa. Roma. pp. 95-111.

Cámara Muñoz A. (2011). "La búsqueda de una profesión. Giulio Lasso en Bretaña”. in Di Fede M.S., Scaduto F. I Quattro Canti di Palermo. Retorica e rappresentazione nella Sicilia del Seicento. Edizioni Caracol. Palermo. pp. 9-24.

Aricò N. (2012). "Pedro Prado e la fondazione di Carlentini", in Casamento A. (coord.). Fondazioni urbane. Città nuove dal medioevo al Novecento. Edizioni Kappa. Roma. pp.168.

Vesco M. (2013). "Un nuovo assetto per il quartiere della Kalsa nel Cinquecento: l'addizione urbana del piano di porta dei Greci". in Cassata G., De Castro E., De Luca M.M. (coord.). Il quartiere della Kalsa a Palermo. Dalle architetture civili e religiose delle origini alle attuali articolate realtà museali. Regione Siciliana. Palermo. pp. 47-65.

Aricò N. (2014). Una città in architettura. Le incisioni di Francesco Sicuro per Messina. Edizioni Caracol. Messina. pp. 92-95.

Bosch Ballbona J. (2014). "La fortaleza que quiso ser palacio. Noticia de Camillo Camiliani en España (1604)". in Locus Amoenus, 12 (2013-2014). pp. 79-106.

Vesco M. (2014). "Il Castellammare di Palermo; un progetto non realizzato di Pietro Antonio Tomasello da Padova”, in Vesco M. (coord.). Ricostruire. Architettura - Storia - Rappresentazione. 1. Edizioni Caracol. Palermo. pp. 7-30.

Vesco M. (2014b)," L'alluvione di Palermo del 1557 tra rischio idrogeologico, speculazione edilizia e piani di ricostruzione". in Galtarossa M., Genovese L. (coord.). La città liquida-la città assetata: storia di un rapporto di lunga durata. Palombi Editori. Roma. pp. 161-187. 